\title{
Technical Comments
}

\section{Comment on "Mechanics of Combustion Mode Transition in a Direct-Connect Ramjet-Scramjet Experiment"}

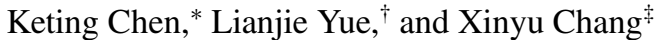 \\ Chinese Academy of Sciences, 100190 Beijing, People's \\ Republic of China
}

DOI: $\underline{10.2514 / 1 . B 36702}$

$\mathbf{I}^{\mathrm{N}}$ N A recent study, Matthew L. Fotia [1] conducted an analysis about the mechanics of combustion mode transition. We appreciate his efforts in trying to shed light on the understanding of discontinuous variation in pressure during the mode transition. Three sets of nonallowable flow configurations in the isolator are identified, and the author claimed that one of them was associated with negative entropy generation based on the second law of thermodynamics analysis of the isolator impulse theory of Heiser and Pratt [1,2]. This is a critical conclusion for the scramjet research, which theoretically demonstrates possible performance mutations during the flight. However, we find that the formula derivation and the physical model used to describe the isolator core flow are not absolutely correct.

For the purpose of discussion and clarity, Eqs. (3-8) quoted in the present Comment are numbered the same as in [1]. And the original equations in [1] have some mistakes; the revised equations are distinguished by an asterisk ("*”) from the original ones.

Considering that no chemical reaction happens and heat transfer is negligible, the isolator flow can be assumed as a steady, adiabatic process with negligible wall friction, which is same as that of Heiser and Pratt [2]. The fluid was treated as calorically perfect gas. The author expressed the differential entropy, static pressure, and Mach number in a one-dimensional compressible flow as

$$
\begin{gathered}
\left.\frac{d s}{c_{p}}=\frac{d T_{0}}{T_{0}}-\frac{\gamma-1}{\gamma} \frac{d P_{0}}{P_{0}}=-\frac{2}{\gamma M^{2}}(1+((\gamma-1) / 2)) M^{2}\right) \frac{d P_{0}}{P_{0}} \\
\frac{d P}{P}=\frac{\gamma M^{2}}{1-M^{2}} \frac{d A}{A}+\frac{2\left(1+((\gamma-1) / 2) M^{2}\right)}{1-M^{2}} \frac{d P_{0}}{P_{0}} \\
\frac{d M^{2}}{M^{2}}=-\frac{2\left(1+((\gamma-1) / 2) M^{2}\right)}{1-M^{2}} \frac{d A}{A} \\
-\frac{2}{\gamma M^{2}} \frac{\left(1+\gamma M^{2}\right)\left(1+((\gamma-1) / 2) M^{2}\right)}{1-M^{2}} \frac{d P_{0}}{P_{0}}
\end{gathered}
$$

In Equations $\left(3^{*}\right)$ and $(4 *)$, some typing or printing mistakes of [1] have been revised according to [3]. However, we doubt the rationality of Eq. (3), which is a basis of the theoretical derivation in [1].

Received 29 March 2017; revision received 1 August 2017; accepted for publication 7 September 2017; published online 27 October 2017. Copyright C) 2017 by the American Institute of Aeronautics and Astronautics, Inc. All rights reserved. All requests for copying and permission to reprint should be submitted to CCC at www.copyright.com; employ the ISSN 0748-4658 (print) or 1533-3876 (online) to initiate your request. See also AIAA Rights and Permissions www.aiaa.org/randp.

*M.E. Student, State Key Laboratory of High-Temperature Gas Dynamics.

'Professor, State Key Laboratory of High-Temperature Gas Dynamics; yuelj@imech.ac.cn (Corresponding Author).

${ }^{\dagger}$ Professor, State Key Laboratory of High-Temperature Gas Dynamics.
A correlation, expressed as Eq. (0) in this Comment, is implicit in Eq. (3*).

$$
\frac{d P_{0}}{P_{0}}=-\frac{\gamma M^{2}}{2} \frac{d T_{0}}{T_{0}}
$$

It reflects essentially the differential stagnation pressure in onedimensional frictionless flow with heat addition [3]. Eqs. (4*) and (5) are also derived by substituting Eq. (ㅁ) into Eqs. (1) and (2), which express the differential pressure and Mach number in onedimensional frictionless compressible flow with heat addition [3] .

$$
\begin{gathered}
\frac{d P}{P}=\frac{\gamma M^{2}}{1-M^{2}} \frac{d A}{A}-\frac{\gamma M^{2}\left(1+((\gamma-1) / 2) M^{2}\right)}{1-M^{2}} \frac{d T_{0}}{T_{0}} \\
\frac{d M^{2}}{M^{2}}=-\frac{2\left(1+((\gamma-1) / 2) M^{2}\right)}{1-M^{2}} \frac{d A}{A} \\
+\frac{\left(1+\gamma M^{2}\right)\left(1+((\gamma-1) / 2) M^{2}\right)}{1-M^{2}} \frac{d T_{0}}{T_{0}}
\end{gathered}
$$

In fact, once Eq. (0) is applied, the core flow has been considered as compressible flow in a variable-area duct with heat addition, which is in conflict with the isolator flow physical process and the assumption of Heiser and Pratt's theory. Eq. (0) seems to be not applicable for the isolator flow. Therefore, Eqs. $\left(3^{*}\right),\left(4^{*}\right)$, and ( $\left.\underline{5}\right)$ are not feasible to describe the adiabatic flow in the isolator.

Besides, there are other errors in the theoretical derivation of negative entropy generation in [1]. As to an adiabatic isolator flow, the change in entropy should be restricted as $d s / c_{p} \geq 0$, according to the second law of thermodynamics. By combining Eqs. $(3 *),(4 *)$, and (5), Fotia [1] claimed that a condition was created that requires

$$
\frac{d P}{P}+\frac{d M^{2}}{M^{2}} \geq-\frac{\left(2-M^{2}\right)}{\left(1-M^{2}\right)} \frac{d A}{A}
$$

However, Eq. (6) is able to correctly represent the constraint of entropy change only for $M<1$, because the correct derivation result of Eq. (6) ought to be

$$
\left(1-M^{2}\right)\left(\frac{d P}{P}+\frac{d M^{2}}{M^{2}}\right)+\left(2-M^{2}\right) \frac{d A}{A} \geq 0
$$

Because the flow in the isolator can be supersonic or subsonic, the term " $1-M^{2}$ " also can be positive or negative. In this regard, if inequality $\left(6^{*}\right)$ is divided by $1-M^{2}$ on both sides, the inequality sign may change when the flow Mach number is less than 1.0. That is to say, inequality (6) is not quite proper for being used to describe the constraint, $d s / c_{p} \geq 0$.

Fotia [1] further derived a more appropriate form of the constraint by introducing the stagnation pressure as

$$
\frac{d P_{0}}{P_{0}}=\frac{d P}{P}+\frac{\gamma M^{2}}{2+(\gamma-1) M^{2}} \frac{d M^{2}}{M^{2}}
$$

Substituting Eq. (7) into inequality (6), he rewrote the constraint as

$$
\frac{\gamma M^{2}}{\left(1-M^{2}\right)} \frac{P}{A}-\left(\frac{d P}{d A}+\Omega_{\text {loss }}\right) \geq 0
$$

where the loss of stagnation pressure is contained in the $\Omega_{\text {loss }}$ term. 


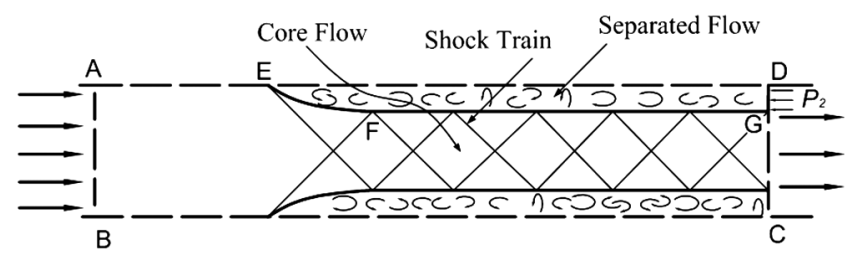

Fig. 1 Control volume for analysis of isolator with confined or separated flow at exit [2].

However, considering that inequality (6) is not a feasible description, the validity of inequality (8) can also be questionable. In fact, the correct form can be attained by substituting Eq. (7) into inequality $(\underline{6 *})$, and it reads

$$
\begin{aligned}
& \left(1-M^{2}\right)\left[\left(2+(\gamma-1) M^{2}\right) \frac{d P_{0}}{P_{0}}+\left(M^{2}-2\right) \frac{d P}{P}\right]+\gamma M^{2}\left(2-M^{2}\right) \frac{d A}{A} \\
& \quad \geq 0
\end{aligned}
$$

For ease of comparison between inequality (8) and inequality ( $\left.8^{*}\right)$, $\Omega_{\text {loss }}$ is used to replace the term of $d P_{0} / P_{0}$ in inequality ( $8 *$ ); thus it can be rewritten as

$$
\left(1-M^{2}\right)\left(2-M^{2}\right)\left(\frac{d A}{P} \Omega_{\text {loss }}-\frac{d P}{P}\right)+\gamma M^{2}\left(2-M^{2}\right) \frac{d A}{A} \geq 0
$$

Inequality (8) in [1] can be generated by dividing both sides of inequality $\left(8^{* *}\right)$ by $\left(1-M^{2}\right)\left(2-M^{2}\right) d A / P$. Nevertheless, the inequality sign may change because the sign of the term $1-M^{2}$ may change at certain flow Mach number. Moreover, according to Heiser and Pratt's isolator relation, Eqs. (1) and (2) in [1], the change in the core flow area $A$ shows a quadratic form with decreasing isolator exit Mach number $M_{2}$, and the area reaches a minimum value at a specific isolator exit Mach number. Thus, the sign of $d A$ will also vary with the isolator exit Mach number. Note that, for given $M_{1}=2.2$, and $\gamma=1.34$, the sign of $d A$ changes at $M_{2}=1.30$. And with an eye on the fact that the term $\left(1-M^{2}\right)\left(2-M^{2}\right) d A / P$ also changes its sign at $M_{2}=1.00$, it is clear how the negative entropy region $\left(1.00<M_{2}<1.30\right)$ was derived incorrectly. Therefore, we think that the negative entropy region, as shown in Fig. 5 in [1], does not exist within the exit Mach number $M_{2}$ range of 1.0-1.3.

In Heiser and Pratt's isolator model, the exit flow must be a confined core flow surrounded by a region of separated flow for any imposed back pressure in the admissible range. The whole straight isolator $\mathrm{ABCD}$ is chosen as the control volume for the analysis, as shown in Fig. 1. The pressure is uniform across the exit crosssectional plane, which is a reasonably good assumption for supersonic compression, and the mean axial velocity in the separated region is either zero or negligible [2]. Let us consider the core flow and separated region separately. For the control volume of the separated flow region DEFG, the impulse function, $I=\dot{m} u+P A$, is $P_{2} A_{\mathrm{DG}}$ at the exit DG and $\int P \mathrm{~d} A$ along the dividing curve EFG. The separated flow exit pressure $P_{2}$ is greater than the upstream pressure for an isolator flow with imposed back pressure. Hence, under the assumption of negligible wall friction, the momentum exchange between the separated flow and the core flow should be taken into account to make the separated flow obey the conservation law of momentum. In other words, it means that there should be viscous shear force between the separated flow and the core flow in the isolator model. Therefore, it may be reasonable to assume a flow in a variable-area duct with friction to describe the core flow in the isolator.

As to an adiabatic flow in a variable-area duct with friction, the differential entropy, static pressure, and Mach number can be expressed as

$$
\begin{gathered}
\frac{d s}{c_{p}}=\frac{d T_{0}}{T_{0}}-\frac{\gamma-1}{\gamma} \frac{d P_{0}}{P_{0}}=-\frac{\gamma-1}{\gamma} \frac{d P_{0}}{P_{0}} \\
\frac{d P}{P}=\frac{\gamma M^{2}}{1-M^{2}} \frac{d A}{A}-\frac{\gamma M^{2}\left[1+(\gamma-1) M^{2}\right]}{2\left(1-M^{2}\right)} \cdot 4 f \frac{d x}{D}
\end{gathered}
$$

$\frac{d M^{2}}{M^{2}}=-\frac{2\left(1+((\gamma-1) / 2) M^{2}\right)}{1-M^{2}} \frac{d A}{A}+\frac{\gamma M^{2}\left(1+((\gamma-1) / 2) M^{2}\right)}{1-M^{2}} \cdot 4 f \frac{d x}{D}$

where $f$ represents nondimensional friction coefficient, and $D$ represents hydraulic diameter.

And since

$$
\frac{d P_{0}}{P_{0}}=-\frac{\gamma M^{2}}{2} \cdot 4 f \frac{d x}{D}
$$

differential static pressure and Mach number can be rewritten as

$$
\begin{gathered}
\frac{d P}{P}=\frac{\gamma M^{2}}{1-M^{2}} \frac{d A}{A}+\frac{1+(\gamma-1) M^{2}}{1-M^{2}} \frac{d P_{0}}{P_{0}} \\
\frac{d M^{2}}{M^{2}}=-\frac{2\left(1+((\gamma-1) / 2) M^{2}\right)}{1-M^{2}} \frac{d A}{A}-\frac{2\left(1+((\gamma-1) / 2) M^{2}\right)}{1-M^{2}} \frac{d P_{0}}{P_{0}}
\end{gathered}
$$

Combining Eqs. (9), (13), and (14) and considering the constraint $d s / c_{p} \geq 0$, a new condition can be obtained according to the aforementioned derivation process, which is the same as Eq. $\left(8^{*}\right)$ or Eq. ( $\left.8^{* *}\right)$.

To summarize, the negative entropy region was achieved by incorrect formula derivation. Furthermore, the physical model of compressible flow in a variable-area duct with heat addition, which was implicitly employed to analyze the isolator flow, is unjustified. But in-depth analysis on the isolator flow can be expected, and thanks are still given for the author's efforts in [1].

\section{References}

[1] Fotia, M. L., "Mechanics of Combustion Mode Transition in a DirectConnect Ramjet-Scramjet Experiment," Journal of Propulsion and Power, Vol. 31, No. 1, 2015, pp. 69-78. doi:10.2514/1.B35171

[2] Heiser, M. L., and Pratt, D. T., Hypersonic Airbreathing Propulsion, Education Series, AIAA, Washington, D.C., 1994, Chap. 6.

[3] Zucrow, M. J., and Hoffman, J. D., Gas Dynamics, Vol. I, Wiley, 1976, pp. $263,315$.

C. Segal Associate Editor 